\title{
Simulation of Electronic Circular Dichroism with Rigid Kohn-Sham Orbitals: A Computational Experiment
}

\author{
Petr Bouř* \\ Institute of Organic Chemistry and Biochemistry, Academy of Sciences of the Czech Republic, Flemingovo nam \\ 2, 16610, Praha 6, Czech Republic
}

Received: June 9, 1998; In Final Form: September 9, 1998

\begin{abstract}
Electronic circular dichroism spectra transition energies and intensities were modeled using the independent particle model within the Kohn-Sham theory. The data obtained were compared with higher level calculations and experimental data. Since the model describes main features in the experimental spectra qualitatively well, it is being proposed as a fast and efficient simulation technique for systems which cannot be dealt with by more sophisticated computations. The best results were obtained with the common B3LYP hybrid density functional.
\end{abstract}

\section{Introduction}

Although a general theory of excited electronic states has not yet been formulated within density functional theory (DFT), the demand for it is apparent from numerous studies published lately. Two distinct approaches have been followed in recent works: the coupled, time-dependent adiabatic extension of DFT (TDDFT $)^{1-3}$ and the perturbation approach based on oneelectron excitations in the Kohn-Sham (KS) determinant. ${ }^{4,5}$ While the former method is generally more accurate, the latter is more universal, computationally faster, and easier to implement. Some studies indicate that KS orbital energy differences are much closer to real excitation energies than those obtained with the Harthree-Fock (HF) determinant. ${ }^{4}$ Perhaps, some modification of the independent particle approximations (IPA) can be justified theoretically within the framework of DFT in the future.

The rigid KS (IPA, DFT-IPA) excitation scheme has often been used in conjunction with the sum-over-states (SOS) expansions. Polarizabilities ${ }^{6}$ as well as tensors needed for nuclear magnetic shielding,, 5 vibrational circular dichroism, ${ }^{8,9}$ and Raman optical activity ${ }^{10}$ could be calculated with comparable accuracy but faster than by conventional coupled-perturbed (CP) methods. Lately, we also used the scheme for calculation of the spin-spin nuclear coupling, ${ }^{11}$ where both the singlet and triplet states are modeled by the KS determinant; nevertheless, the approach was not accurate enough to sense singlet/triplet energy differences.

In this study, the IPA-KS scheme is applied to calculation of electronic circular dichroism (ECD) spectra as an empirical method. Unlike for the applications mentioned in the previous paragraph, the ECD intensities directly reflect individual electron transitions, i.e., their energies and charge distributions. This enables a more detailed insight into the properties of the KS determinant. Since such a calculation of ECD requires a negligible increase of computational time compared to a singlepoint energy calculation, it may provide fast qualitative estimates of electronic optical activity for considerably larger molecules * Corresponding author. E-mail: bour@uochb.cas.cz. Fax (420-2)-2431-
0503 .

10.1021/jp982550d CCC: $\$ 18.00$ ㄷ 1999 American Chemical Society than are presently the case. ECD is generally difficult to predict using ab initio calculations, ${ }^{12,13}$ and such an extension of the theory may be immediately applied to numerous systems.

\section{Calculations}

In the present approximation, the excitation energies are equal to the difference of Kohn-Sham orbital energies, $\epsilon_{J}-\epsilon_{K}$. This simplification substantially increases flexibility of "ab initio" studies of molecular properties since the single-point calculation of the KS determinant can be performed easily. Three common DFT functionals are investigated: the local spin density approximation $(\mathrm{LDA}, \mathrm{LSDA}=\mathrm{SVWN}),{ }^{14}$ the gradient-corrected correlation functional of Becke ${ }^{15}$ with the exchange functional of Perdew and Wang ${ }^{16}$ (BPW91), and finally, the threeparameter functional of Becke (B3LYP). ${ }^{17}$

Molecular geometries were optimized using the Gaussian ${ }^{18}$ program. The output of Gaussian, i.e., orbital energies and coefficients, was analyzed with the Roa program ${ }^{10}$ written in house. The program Turbomole ${ }^{3,19}$ was used for the timedependent DFT calculations; a more time-demanding TDDFT implementation in Gaussian provided similar results for control tasks.

An electronic transition $n \rightarrow j$ can be observed in a CD spectrum as a band with an area proportional to the optical rotational strength, $R$,

$$
R_{n \rightarrow j}=\operatorname{Im}\langle n|\boldsymbol{\mu}| j\rangle \cdot\langle j|\mathbf{m}| n\rangle
$$

where $\boldsymbol{\mu}$ and $\mathbf{m}$ are the electric and magnetic moments, respectively. ${ }^{20}$ The origin dependence of the results was neglected for our systems with coordinate origins near geometrical centers.

\section{Results and Discussion}

BH and $\mathbf{C H}_{2}$. For these two molecules, results obtained by the IPA and the B3LYP functional are compared to TDDFT and full $\mathrm{CI}(\mathrm{FCI})^{21}$ calculations. The standard $6-311 \mathrm{G}^{* *}$ basis of Gaussian corresponds approximately to the TZV basis of Turbomole; the cc-pVTZ Dunning's correlation consistent basis 
TABLE 1: Excitation Energies for $\mathrm{BH}^{a}{ }^{a}$ in $\mathbf{~ n m}$

\begin{tabular}{|c|c|c|c|c|c|c|c|}
\hline \multirow[b]{2}{*}{ state } & \multicolumn{2}{|c|}{ IPA } & \multicolumn{2}{|c|}{ TDDFT } & \multicolumn{2}{|c|}{ CIS } & \multirow[b]{2}{*}{$\mathrm{FCI}^{21}$} \\
\hline & $6-311 G^{* *}$ & cc-pVTZ & $\mathrm{TZV}$ & $\begin{array}{c}\text { cc-pVTZ } \\
\text { (triplet) }\end{array}$ & $6-311 \mathrm{G}^{* *} *$ & cc-pVTZ & \\
\hline${ }^{1} \Pi$ & 314 & 315 & 456 & $460(467)$ & 426 & 430 & 421 \\
\hline${ }^{1} \Sigma$ & 138 & 140 & 177 & $178(184)$ & 137 & 149 & 194 \\
\hline${ }^{1} \Pi$ & 115 & 116 & 137 & $136(131)$ & 107 & 117 & 166 \\
\hline${ }^{1} \Pi$ & 87 & 107 & 118 & $123(126)$ & 92 & 106 & 150 \\
\hline${ }^{1} \Sigma$ & 99 & 121 & 137 & 137 (157) & 101 & 125 & 177 \\
\hline${ }^{1} \Sigma$ & 87 & 112 & 117 & $119(151)$ & 86 & 107 & 164 \\
\hline
\end{tabular}

TABLE 2: Excitations in $\mathrm{CH}_{2}{ }^{a}$

\begin{tabular}{|c|c|c|c|c|c|c|c|c|}
\hline \multirow[b]{3}{*}{ state } & \multicolumn{7}{|c|}{ (A) Energies, in $\mathrm{nm}$} & \multirow[b]{3}{*}{$\mathrm{FCI}^{21}$} \\
\hline & \multicolumn{2}{|c|}{ IPA } & \multicolumn{2}{|c|}{ TDDFT } & \multicolumn{3}{|c|}{ CIS } & \\
\hline & $6-311 \mathrm{G}^{* *}$ & cc-pVTZ & TZV cc & $c-p V T Z \quad 6-3$ & $-311 G^{* *}$ & cc-pV & $J \mathrm{TZ}$ & \\
\hline${ }^{1} \mathrm{~A}_{1}$ & 153 & 154 & 184 & 173 & 154 & 15 & & 266 \\
\hline${ }^{1} \mathrm{~A}_{1}$ & 82 & 87 & 114 & 111 & 81 & 8 & 9 & 190 \\
\hline${ }^{1} \mathrm{~A}_{1}$ & 78 & 84 & 99 & 99 & 78 & 88 & & 146 \\
\hline${ }^{1} \mathrm{~B}_{1}$ & 129 & 128 & 147 & 141 & 129 & 128 & & 161 \\
\hline${ }^{1} \mathrm{~B}_{1}$ & 92 & 93 & 112 & 110 & 93 & 9 & 5 & 155 \\
\hline${ }^{1} \mathrm{~B}_{2}$ & 428 & 422 & 1995 & 1190 & 1012 & 99 & & 691 \\
\hline${ }^{1} \mathrm{~B}_{2}$ & 83 & 83 & 112 & 109 & 74 & 8 & 9 & 139 \\
\hline${ }^{1} B_{2}$ & 69 & 84 & 81 & 87 & 71 & 7 & 4 & 117 \\
\hline${ }^{1} \mathrm{~A}_{2}$ & 149 & 151 & 194 & 200 & 180 & 18 & & 212 \\
\hline \multicolumn{9}{|c|}{ (B) Oscillator Strengths } \\
\hline & \multicolumn{2}{|c|}{ IPA } & \multicolumn{2}{|c|}{ TDDFT } & \multicolumn{4}{|c|}{ CIS } \\
\hline state & $6-311 \mathrm{G}^{*}$ & cc-pVTZ & $\mathrm{TZV}$ & cc-pVTZ & $6-311$ & $\mathrm{G}^{* *}$ & cc-p & pVTZ \\
\hline${ }^{1} \mathrm{~A}_{1}$ & 0.06 & 0.06 & 0.10 & 0.10 & 0.1 & & & .11 \\
\hline${ }^{1} \mathrm{~A}_{1}$ & 0.09 & 0.00 & 0.01 & 0.03 & 0.1 & & & .07 \\
\hline${ }^{1} \mathrm{~A}_{1}$ & 0.00 & 0.06 & 0.18 & 0.16 & 0.0 & & & .18 \\
\hline${ }^{1} \mathrm{~B}_{1}$ & 0.06 & 0.07 & 0.08 & 0.08 & 0.0 & & & .09 \\
\hline${ }^{1} \mathrm{~B}_{1}$ & 0.20 & 0.20 & 0.15 & 0.14 & 0.2 & & & .01 \\
\hline${ }^{1} \mathrm{~B}_{2}$ & 0.01 & 0.01 & 0.01 & 0.00 & 0.0 & & & .01 \\
\hline${ }^{1} \mathrm{~B}_{2}$ & 0.24 & 0.24 & 0.39 & 0.16 & 0.3 & & & .32 \\
\hline${ }^{1} \mathrm{~B}_{2}$ & 0.01 & 0.00 & 0.02 & 0.03 & 0.1 & & & .03 \\
\hline${ }^{1} \mathrm{~A}_{2}$ & 0 & 0 & 0 & 0 & 0 & & 0 & ) \\
\hline
\end{tabular}

includes also a set of $d$ orbitals on the hydrogen and $\mathrm{f}$ orbitals on heavy atoms. In Table 1, six lowest singlet excitation energies for $\mathrm{BH}$ are listed. The best approximation of the FCI lowest excitation energy $(421 \mathrm{~nm})$ was obtained with the CIS model. The TDDFT values are, however, more consistent and accurate on average. Except of the first transition, the IPA values are remarkably close to the CIS numbers, which can explain the successes of the rigid scheme mentioned in the Introduction. Clearly, the quality of the basis plays a minor role in the accuracy of the lowest energy transitions, within the error bars pursued in this work. The difference between the singlet and triplet transition energies (indicated for the TDDFT calculation) is smaller than the overall error. For BH, the singlet/triplet corrections apparently do not affect the ordering of the first four transitions. Note that, for most molecules, ECD spectra comprise just the few lowest energy singlet transitions.

For the $\mathrm{CH}_{2}$ molecule, the energies and oscillator strengths are collected in Table 2. Similarly to the case for BH, the IPA energies closely follow the CIS numbers, with the exception of the first ${ }^{1} \mathrm{~B}_{2}$ transition. The energy of this transition is also purely matched by the TDDFT value; nevertheless, the TDDFT method again gives the best results on average. Greater variations with respect to the basis sets and approximations can be observed for the oscillator strengths, although relative band intensities are reasonably reproduced by all methods.

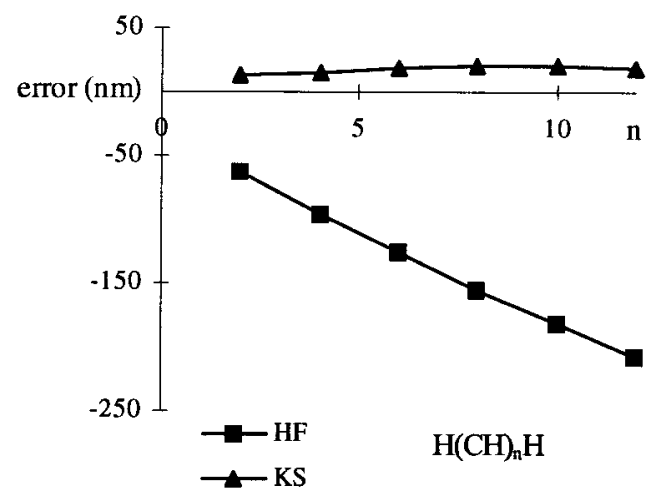

Figure 1. Calculated difference between the LUMO-HOMO gap and the first singlet excitation energy, as a function of molecular size for polyacetylene.

At this point, one can conclude that the IPA with the B3LYP functional provides excitation energies comparable to the classical CIS calculation, is much faster, and is relatively insensitive to small changes in the basis set quality but is inferior to the TDDFT method. Obviously, these conclusions cannot be extended to systems with more complicated electron configurations (high symmetry, avoided crossings) without further testing.

$\mathbf{H}-(\mathbf{C H})_{n}-\mathbf{H}$. Since bigger molecules are the primary targets of the ECD simulations, the influence of molecular size on the stability of IPA excitation energies was tested for polyacetylene. Geometries of $\mathrm{H}-(\mathrm{CH})_{n}-\mathrm{H}(n=2,4,6,8,10$, and 12) were optimized $(\mathrm{HF} / 6-31 \mathrm{G})$ for the all-trans planar arrangement. Then, the HOMO-LUMO gap obtained by the HF and KS/ B3LYP theories was compared to the lowest singlet excitation energy calculated with the TDDFT method. The error, equal to the difference between the gap and the TDDFT energy, was plotted as a function of molecular size. Striking contrast between the $\mathrm{HF}$ and $\mathrm{KS}$ computations is apparent in Figure 1. While the HF error is already greater than $50 \mathrm{~nm}$ for ethylene and reaches $208 \mathrm{~nm}$ for $n=12$, the KS HOMO-LUMO gap deviates from the reference by about $20 \mathrm{~nm}$, which would be an acceptable value for a comparison with an experiment. Moreover, the error for the KS-IPA scheme is almost independent of molecular size.

$(2 S, 3 S)$ - $N$-Chlor- $(2,3)$-dimethylaziridine (A) and $(2 S, 3 S)$ $\mathrm{N}$-Brom-(2,3)-dimethylaziridine (B). Optically active aziridines

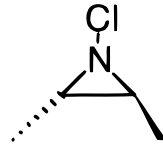

A

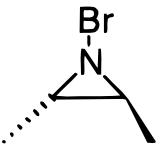

B have many practical applications, and ECD spectroscopy may be conveniently used for assigning their absolute stereochemistry. ${ }^{13}$ The halogen substitution on the nitrogen results in a welldefined chromophore for which the ECD spectrum and hence the absolute configuration can be reliably measured. Here, the IPA calculation of ECD is compared to experimental parameters, previous CIS calculations, and the TDDFT theory.

Equilibrium geometries were obtained with the B3LYP functional and $6-31+G^{*}\left(6-311 G^{*}\right.$ for B) basis. In Figure 2, the whole IPA-ECD pattern was simulated for $\mathbf{A}$ using the HF Hamiltonian, B3LYP, BPW, and LDA approximation. Gaussian bands with a bandwidth of $10 \mathrm{~nm}$ were used for simulated spectra, roughly corresponding to the experimental conditions. Apparently, the HF transitional energies are substantially lower 
TABLE 3: ECD Parameters for the Aziridin Derivatives ${ }^{a}$

\begin{tabular}{|c|c|c|c|c|c|c|c|c|c|c|c|}
\hline \multirow[b]{2}{*}{$\operatorname{trans}$} & \multicolumn{4}{|c|}{$\lambda$} & \multicolumn{3}{|c|}{$\mu$} & \multicolumn{4}{|c|}{$R$} \\
\hline & CIS $^{13}$ & IPA & TDDFT & $\exp ^{13}$ & CIS & IPA & TDDFT & $\mathrm{CIS}^{13}$ & IPA & TDDFT & $\exp ^{13}$ \\
\hline \multicolumn{12}{|c|}{ A } \\
\hline$\pi^{*}-\sigma^{*}$ & 206 & 220 & 278 & 260 & 0.87 & 1.02 & 0.70 & -1.7 & -4.8 & -2.4 & -3.2 \\
\hline $\mathrm{p}-\sigma^{*}$ & 178 & 185 & 227 & 212 & 0.31 & 0.26 & 0.29 & -0.7 & 2.7 & 1.6 & $2.9^{b}$ \\
\hline \multicolumn{12}{|c|}{ B } \\
\hline$\pi^{*}-\sigma^{*}$ & & 249 & 329 & 303 & & 1.2 & 0.78 & & -7.3 & -3.4 & -5.1 \\
\hline $\mathrm{p}-\sigma^{*}$ & & 214 & 271 & 249 & & 0.23 & 0.26 & & 1.12 & 0.8 & $4.0^{b}$ \\
\hline
\end{tabular}

${ }^{a}$ Wavelength $(\lambda)$ is given in $\mathrm{nm}$, transition dipole moment $(\mu)$ in debyes, and rotational strength $(R)$ in $10^{-40}$ cgs units. ${ }^{b}$ Rough estimation.

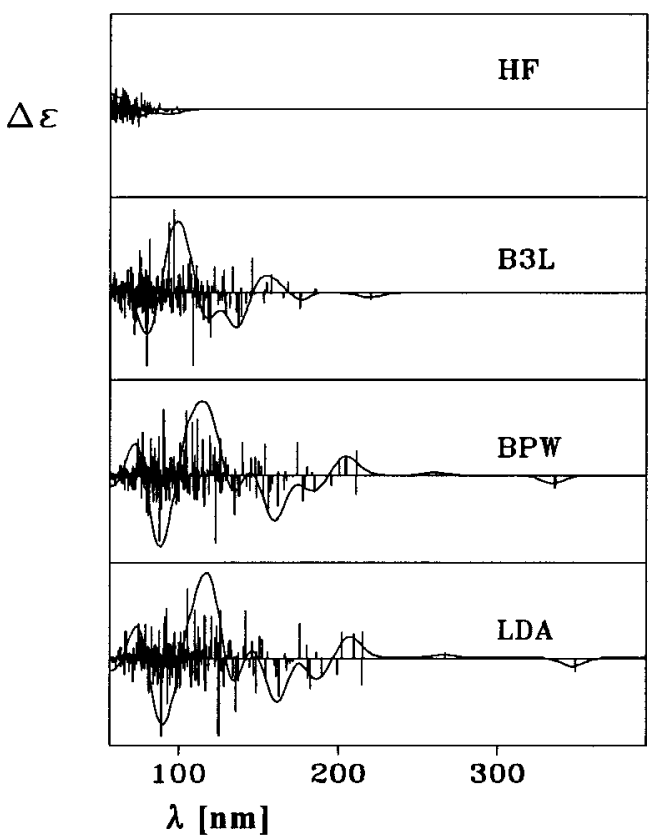

Figure 2. ECD spectra of A simulated using the HF, B3LYP, BPW91, and LDA orbitals and the IPA. The spectra are plotted in the same scale.

than the experimental ones and the order of the transitions is incorrect, as far as can be judged from the spectral pattern. On the other side, the last two BPW91 and LDA approximations lead not only to the true order of the two observed lowest energy singlet transitions but also to correct signs and relative magnitudes of the CD bands. These pure-DFT schemes overestimate the transitional wavelengths, and thus the hybrid "compromised" B3LYP functional yields the best spectrum. Interestingly, calculated spectral DFT patterns are relatively independent of the functional throughout the frequency region, which gives some credibility also to the far-UV part of the spectrum that can be measured and interpreted so rarely.

Calculated energies and dipolar and rotational strengths are summarized in Table 3. As apparent, the CIS energies deviate most from the experiment. This method even predicts the wrong sign for the $\mathrm{p}-\sigma^{*}$ transition in $\mathbf{A}$ (CIS calculation of $\mathbf{B}$ was not feasible in ref 13 because of the size of the molecule). The IPA and TDDFT calculations provide more realistic magnitudes of rotational strengths and correctly describe relative magnitudes of the $\pi^{*}-\sigma^{*}$ and $\mathrm{p}-\sigma^{*}$ transitions as well as the red shift and increase of $\mathrm{CD}$ intensity observed upon substitution with the bromine. The TDDFT calculation yielded energies about $20 \mathrm{~nm}$ higher than the experimental; the IPA values are lower by about 30-45 nm. The IPA yielded correct spectral patterns also for other small molecules (not shown here), and thus, because of its simplicity, it may be recommended as a starting point for more sophisticated ab initio simulations of ECD.

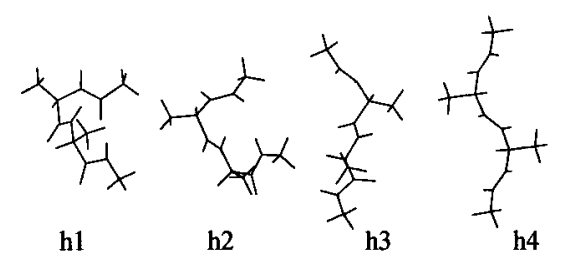

Figure 3. Conformations of ( $N$-acetyl-L-alanyl)-L-alanine methylamid mimicking $\alpha$-helix (h1, $\left.\phi=-57^{\circ}, \psi=-47^{\circ}\right), 3-10$ helix (h2, $\phi=$ $\left.-60^{\circ}, \psi=-30^{\circ}\right)$, polyproline II $\left(\mathbf{h 3}, \phi=-78^{\circ}, \psi=149^{\circ}\right)$, and $\beta$-sheet $\left(\mathbf{h} 4, \phi=-128^{\circ}, \psi=124^{\circ}\right)$.

ECD of Peptides. Unlike for the smaller molecules, simulation of peptide ECD can be encountered rarely in the literature, although conformational studies of peptides and proteins have become a natural area of interest in CD spectroscopy. The amide group, peptides' main chromophore, absorbs in the far-UV region on the edge of the instrumentally available region, strongly interacts with the solvent, and is influenced by the conformation and structure of the amino acid side chains. Nevertheless, the dependence of the spectra on the conformation was recognized empirically and can be even used for quantitative predictions. $^{22}$

To investigate a basic dependence of the ECD pattern on the peptide main chain conformation, a model system was chosen consisting of four conformations of $N$-(N-acetyl-L-alanyl)-Lalanine methylamid corresponding to $\alpha$-helix (h1), 3-10 helix (h2), polyproline II conformation (h3), and $\beta$-sheet (h4) as indicated in Figure 3. The geometries were optimized (BPW91/ $631 \mathrm{G}^{* *}$ ) under constraints for the main chain torsion angles $(\omega, \phi, \psi)$. The polyproline II conformation is supposedly similar to the "random" conformation of oligopeptides. ${ }^{23}$ Average torsion angles for parallel and antiparallel strands were assigned to $\beta$-sheet. The amide group was constrained to a planar trans arrangement $\left(\omega=180^{\circ}\right)$. Since absorption bands of aliphatic amino acid side chains do not interfere with the measured spectrum, the methyl group used in the model may be considered a reasonable approximation.

In Figure 4, simulated spectra (with the IPA and TDDFT methods, both with the B3LYP/6-31G** basis) are compared to standard spectra of poly-L-lysine conformations. ${ }^{24}$ Many interesting links between the calculated and experimental spectra can be found. For example, the two negative peaks found by experiment at 222 and $209 \mathrm{~nm}$ for the $\alpha$-helix (h1) are reproduced by the IPA calculation at 203 and $195 \mathrm{~nm}$, respectively; the TDDFT model yields only one negative band at $236 \mathrm{~nm}$. Also, the positive sign of the experimental band at $193 \mathrm{~nm}$ is reproduced correctly by both the IPA and TDDFT models (at 186 and $228 \mathrm{~nm}$ ). The lower wavelength minima predicted at 198-210 nm (TDDFT) and 167-177 nm (IPA, see also Figure 5, where a broader range is captured for h1) match the observed minimum at about $160 \mathrm{~nm}$ of poly- $\gamma$ methylglutamate ${ }^{25}$ and perhaps also the observed positive signal below $150 \mathrm{~nm}$ can be associated with the computations. 


\section{Calculation}

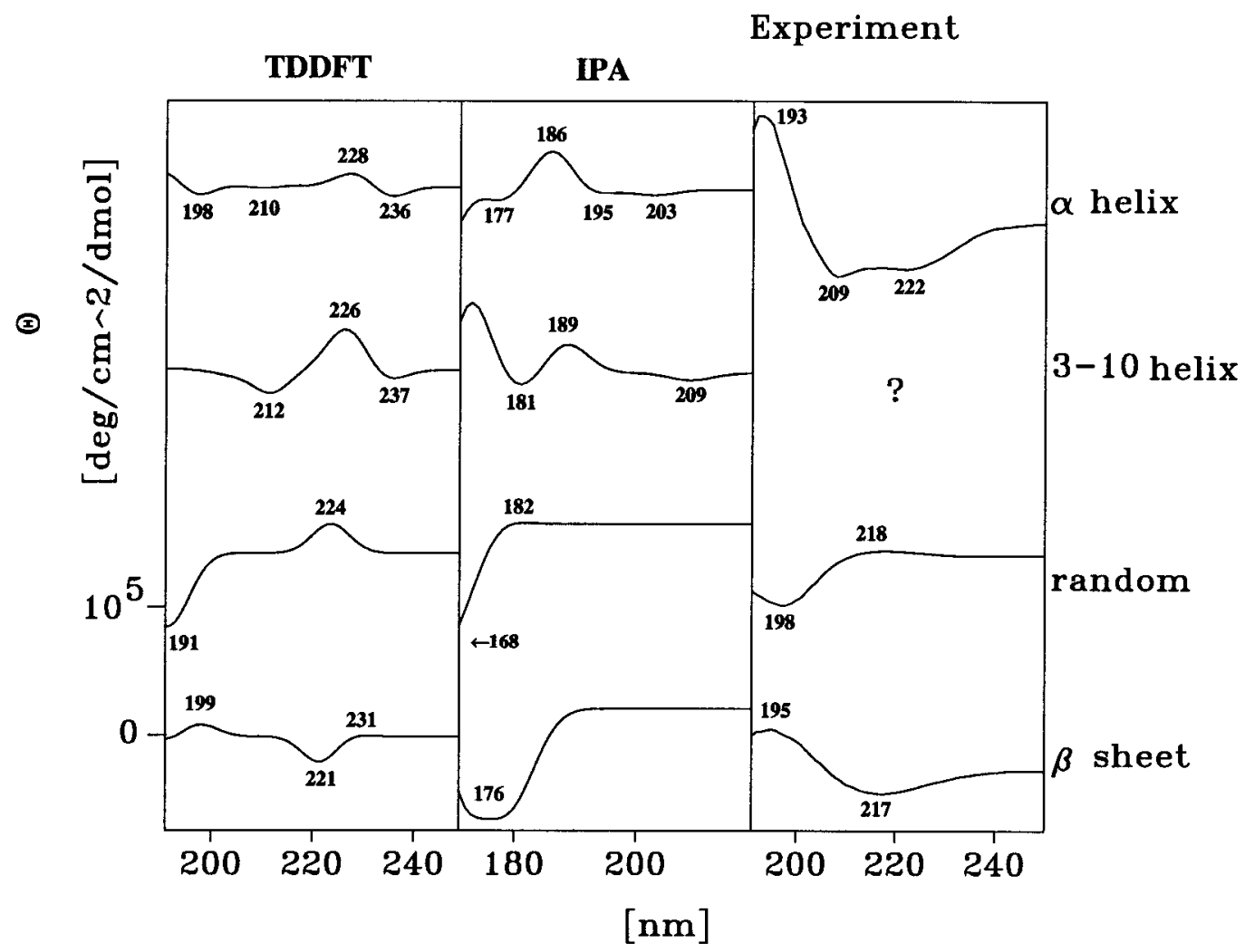

Figure 4. Simulated and experimental spectra of the four peptide conformations.

However, for this region, an interference of the amino acid side chain can contribute unpredictably to the spectrum.

For the experimentally not well-established spectrum of the 3-10 helix (h2), the two models predict a red shift of the lowest energy negative band and a relatively bigger change of intensity of the bands at lower wavelengths.

Upon the conformational change from $\alpha$-helix to the random conformation (h3), a blue shift of 12 and $21 \mathrm{~nm}$ is predicted for the highest wavelength band by the TDDFT and IPA models, respectively. This overestimates the shift of $4 \mathrm{~nm}$ corresponding to the small maximum observed at $218 \mathrm{~nm}$ in the spectra of poly-L-lysine. Calculated signs of the negative band also correspond to the experiment, but predicted positions do not correlate with the experimental value of $198 \mathrm{~nm}$.

Finally, for $\beta$-sheet (h4) experimental wavelength of the highest frequency band $(217 \mathrm{~nm})$ is close to the $218 \mathrm{~nm}$ band of the random conformation, and its sign is negative. These features are reproduced by both theoretical predictions. Additionally, the TDDFT theory also predicts a positive signal at $231 \mathrm{~nm}$, which may reflect the small positive band in this region observed in some experimental studies of $\beta$-sheets. ${ }^{26}$

Conformational studies of proteins are hampered by the absence of a model experimental spectrum of 3-10 helix. Nevertheless, as indicated in Figure 5, small variations of the torsion angles cause substantial changes in spectral intensities, and thus a distinct spectrum of this conformation should exist.

Trial calculations (not shown here) for longer peptide segments performed at a lower level indicated a reasonable stability of the ECD spectral pattern with respect to changes of molecular size. This is in accord with experimental observation; that is, it is the peptide conformation rather than its length which ultimately determines the spectral pattern. Relation between

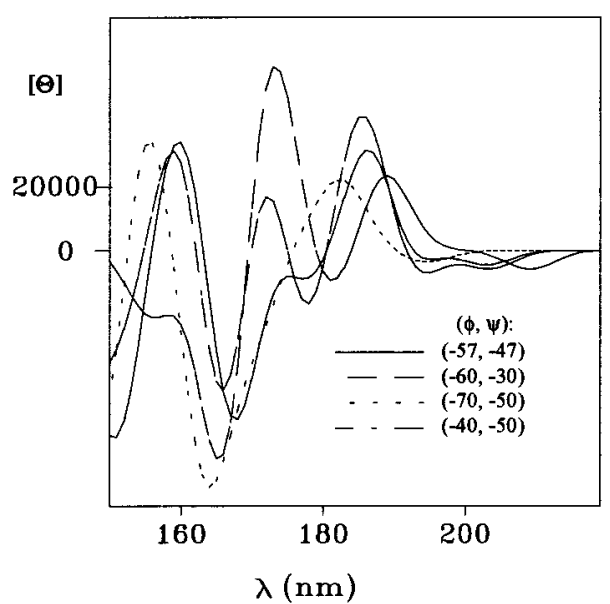

Figure 5. Calculated ECD spectra for four conformations close to $\alpha$-helix.

peptide length and spectral pattern was experimentally established only for $\alpha$-helix,${ }^{27}$ where relative intensity of the lowest energy transition slightly increases for longer molecules.

At this point, it can be concluded that the IPA as well as the TDDFT modeling qualitatively describe the conformational dependence of ECD. Apparently, neither of the two approaches provided quantitatively accurate predictions of absolute ECD intensities. These can be influence by the solvent, dynamical conformational equilibria, and other factors that are difficult to model with ab initio methods. The results (cf. Figure 4) indicate that the IPA model may be more sensitive to conformational changes than the adiabatic theory. Note that only the singlepoint energy had to be calculated for the IPA model, which lasted $38 \mathrm{~min}$ on the Pentium II processor $(400 \mathrm{MHz})$ with the Turbomole program. Significantly longer time was needed for 


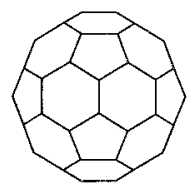

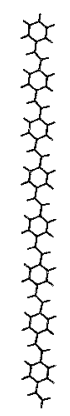

$\lambda[\mathrm{nm}]$
HOMO

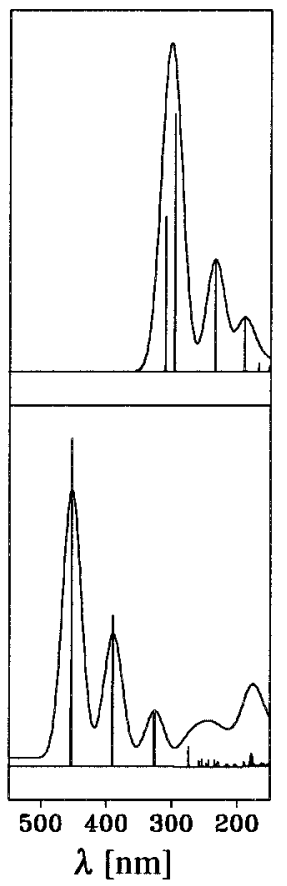

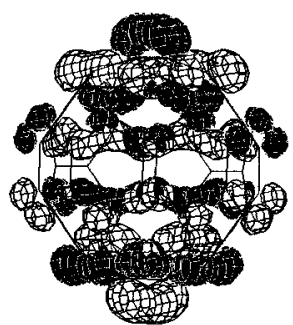

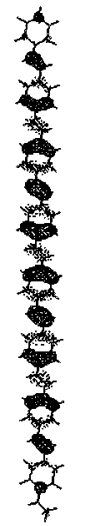

LUMO
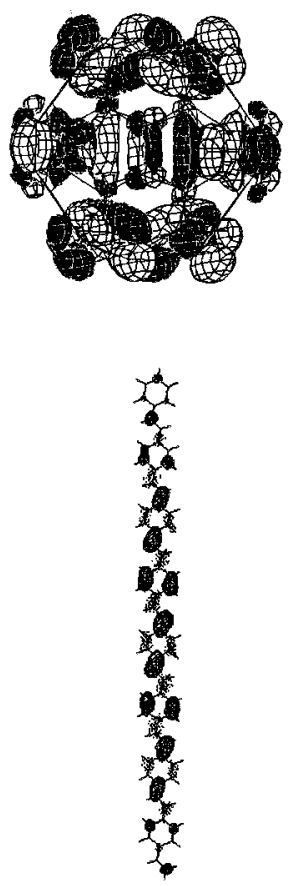

Figure 6. Simulated absorption spectra and boundary orbitals for $\mathrm{C}_{60}$ (up) and $\mathbf{C}$ (down).

TABLE 4: First Two Symmetric Singlet Transitions in C and $\mathrm{C}_{60}{ }^{a}$

\begin{tabular}{llccccc}
\hline & & $\begin{array}{c}\text { basis: } \\
\text { method: }\end{array}$ & $\begin{array}{c}\text { 6-31g } \\
\text { IPA }\end{array}$ & $\begin{array}{c}\text { SV } \\
\text { TDDFT }\end{array}$ & $\begin{array}{c}\text { 6-31g* } \\
\text { IPA }\end{array}$ & $\begin{array}{c}\text { SV(P) } \\
\text { TDDFT }\end{array}$ \\
\hline $\mathbf{C}$ & 1 & & 457 & 517 & 464 & \\
& 2 & & 423 & 452 & 429 & \\
$\mathrm{C}_{60}$ & 1 & & 188 & 213 & 192 & 218 \\
& 2 & & 181 & 198 & 184 & 202
\end{tabular}

${ }^{a}$ Energies in $\mathrm{nm}$. The 6-31g and SV bases are almost identical. Addition of polarization functions, which approximately doubles basis set size, is denoted by * and $(\mathrm{P})$, respectively.

the solution of time-dependent Kohn-Sham equations (5 days for 20 transitions in h1, under the same conditions).

In the past, we used a similar model to simulate vibrational circular dichroism (VCD) of peptides, ${ }^{28}$ which provided a more satisfactory agreement with experiment than the ECD model presented here. Generally, many applications of ECD are problematic because of the lack of reliable simulation and interpretation techniques. This difficulty underlines principal differences between the ECD and VCD phenomena, namely the volatility and complexity of the former and the locality and limited environmental dependence of the latter.

Polymeric Semiconductors. To illustrate further applications, absorption spectra of the fullerene $\left(\mathrm{C}_{60}\right)$ and $\mathrm{Phe}-[\mathrm{CH}=\mathrm{CH}-$ Phe $]_{7}-\mathrm{CH}=\mathrm{CH}_{2}(\mathbf{C})$ molecules were simulated. Such compounds currently attract attention as low-cost semiconductors and superconductors, and theoretical studies of the electronic structure significantly enhance their rational design. ${ }^{29}$ Although not optically active, the HOMO/LUMO gap and the lowest energy excitations determine the most important conductivity properties, similarly as for ECD. The B3LYP/6-31G equilibrium geometry was used for $\mathrm{C}_{60}$, while the structure of $\mathbf{C}$ was obtained via propagation of the optimized (B3LYP/6-31G*) geometry of $\mathrm{Phe}-\mathrm{CH}=\mathrm{CH}-\mathrm{Phe}-\mathrm{CH}=\mathrm{CH}_{2}$. The same B3LYP functional was used for computations of energies of the first two symmetric transitions collected in Table 4 . The size of the basis set is apparently a minor factor for the accuracy of calculated energies, which is rather surprising for systems of this size. The IPA model predicts the wavelength of the lowest energy transition in $\mathbf{C}$ to be higher by $269 \mathrm{~nm}$ than that for $\mathrm{C}_{60}$. This is in accordance with the difference of $304 \mathrm{~nm}$ obtained by the TDDFT scheme and is in agreement with the absorption and conductivity spectra of derivatives of both compounds examined in ref 29. In Figure 6, absorption spectra of the two compounds are simulated using the IPA scheme. The first $\left(\mathrm{HOMO} \rightarrow\right.$ LUMO) transition of $\mathrm{C}_{60}$ calculated at 431 $\mathrm{nm}$ cannot be seen since it is not electrically allowed. It may be visible for a derivative of $\mathrm{C}_{60}$, and its interaction with the first transition of the polymer (calculated at $454 \mathrm{~nm}$ ) may lead to an overall increase of absorption of the mixture of the two compounds, as observed at about $516 \mathrm{~nm}$. Apart from the beauty of the orbitals, their shape, as indicated in Figure 6, may account for many physical properties of the compounds. For instance, the lobes of the LUMO in $\mathbf{C}$ are oriented approximately along the molecular axis, which supposedly enhances electric conductivity. ${ }^{29}$

These results correspond to the study of Salzner at al., ${ }^{30}$ where the HOMO/LUMO gap estimated from KS energies is compared to experiment for semiconductor polymers. This study also indicated the sharp difference in the behavior of HF and KS energies as well as the superiority of the hybrid functionals. Thus, the present work suggests that such calculations of electronic energies based on the KS determinant can be extended for estimation of other molecular properties.

\section{Conclusions}

The calculation of electronic excitations based on the rigid KS orbitals provided fast and relatively accurate predictions of excitation energies and ECD intensities. Typically, the error of energies for the lowest energy transitions was comparable to 
or smaller than that for values obtained by the CIS method. The B3LYP functional led to better results than the LDA and BPW91 approximations. ECD simulations based on the rigid HF orbitals were not found to be meaningful, in accord with previous studies.

Acknowledgment. The work was supported by the Grant Agency of the Czech Republic (Grant 203/97/P002). The author thanks Dr. Radek Pelc for help with the manuscript.

\section{References and Notes}

(1) Casida, M. E.; Jamorski, C.; Casida, K. C.; Salahub, D. R. J. Chem. Phys. 1998, 108, 449.

(2) Jamorski, C.; Casida, M. E.; Salahub, D. R. J. Chem. Phys. 1996, $104,5134$.

(3) Bauernschmitt, R.; Ahlrichs, R. Chem. Phys. Lett. 1996, 256, 454. 9994.

(4) Filippi, C.; Umrigar, C. J.; Gonze, X. J. Chem. Phys. 1997, 107,

(5) Malkin, V. G.; Malkina, O.; Eriksson, L. A.; Salahub, R. D. In Modern Density Functional Theory; Seminario, J. M., Politzer, P., Eds.; Elsevier: Amsterdam, 1995; p 273.

(6) Bouř, P. Chem. Phys. Lett. 1997, 265, 65

(7) Schreckenbach, G.; Dickson, R. M.; Ruiz-Morales, Y.; Ziegler, T. In Chemical Applications of Density-Functional Theory; Laird, B. B., Ross, R. B., Ziegler, T., Eds.; ACS Symposium Series 629; American Chemical Society: Washington, 1996; Chapter 23.

(8) Bouř, P.; McCann, J.; Wieser, H. J. Chem. Phys. 1998, 108, 8782. 9783.

(9) Bouř, P.; McCann, J.; Wieser, H. J. Phys. Chem. A 1997, 101,

(10) Bouř, P. Chem. Phys. Lett. 1998, 288, 363.

(11) Bouř, P.; Buděšínský, M. J. Chem. Phys. 1999, 110, 2836-2843.
(12) Bak, K. L.; Hansen, A. E.; Ruud, K.; Helgaker, T.; Olsen, J.; Jørgensen, P. Theor. Chim. Acta 1995, 90, 441.

(13) Shustov, G. V.; Kachanov, A. V.; Korneev, V. A.; Kostyanovsky, R. G.; Rauk, A. J. Am. Chem. Soc. 1993, 115, 10267. Experimental values for $n$-hexane solution; as follows from a communication with Prof. A. Rauk, calculated rotational strengths in the reference were by a mistake multiplied by a factor of 2 .

(14) Slater, J. C. Quantum Theory of Molecular and Solids, Vol. 4: The Self-Consistent Field for Molecular and Solids; McGraw-Hill: New York, 1974.

(15) Becke, A. D. Phys. Rev. A 1998, 38, 3098.

(16) Perdew, J. P.; Wang, Y. Phys. Rev. B 1992, 45, 13244.

(17) Becke, A. D. J. Chem. Phys. 1993, 98, 5648.

(18) Frisch, M. J., et al. Gaussian 98, revision A.3.; Gaussian, Inc.: Pittsburgh, PA, 1998.

(19) The Encyclopedia of Computational Chemistry; Schleyer, P. v. R., Allinger, N. L., Clark, T., Gasteiger, J., Kollman, P. A., Schaefer, H. F., III; Schreiner, P. R., Eds.; John Wiley \& Sons: Chichester, 1998.

(20) Barron, L. D. Molecular Light Scattering and Optical Activity; Cambridge University Press: Cambridge, 1982.

(21) Christiansen, O.; Koch, H., Jørgensen, P. J. Chem. Phys. 1996, $105,1451$.

(22) Toumadje, A.; Johnson, W. C. Anal. Biochem. 1993, 211, 258

(23) Dukor, R. K.; Keiderling, T. A. Biopolymers 1991, 31, 1747.

(24) Greenfield, N.; Fasman, G. D. Biochemistry 1969, 8, 4108.

(25) Johnson, W. C.; Tinoco, I. J. Am. Chem. Soc. 1972, 94, 4389.

(26) Bolotina I. A. Mol. Biol. (USSR) 1981, 15.

(27) Chen, Y. H.; Yang, J. T. Biochemistry 1974, 13, 3370.

(28) Bouř, P.; Keiderling, T. A. J. Am. Chem. Soc. 1993, 115, 9602.

(29) Köhler, A.; dos Santos, D. A.; Beljonne, D.; Shuai, Z.; Brédas, J. L.; Holmes, A. B.; Kraus, A.; Müllen, K.; Friend, R. H. Nature 1998, 392 903.

(30) Salzner, U.; Lagowski, J. B.; Pickup, P. G.; Poirier, R. A. J. Comput. Chem. 1997, 18, 1943. 